\section{Commentary: Graft flow assessment-Friend, not foe, preventing vertigo and crash}

\author{
Chaim Locker, MD
}

In 2007, during a routine training flight off the Oregon coast, an experienced 34-year-old pilot of the Air National Guard flew his F-15A fighter right into the Pacific Ocean at a speed of more than $600 \mathrm{mph}$. The pilot was killed, and the $\$ 32$ million aircraft was destroyed. ${ }^{1}$

In their article in this issue of the Journal, Akhrass and Bakaeen $^{2}$ provide an excellent, up-to-date review on the current available instruments and knowledge regarding intraoperative graft flow measurements during coronary artery bypass grafting (CABG) surgery. They present important studies on this subject and explore the concept that no definite conclusion can be drawn regarding the absolute yield of those measurements; is it a "friend" or a "foe"? They conclude with a set of recommendations but leave the coronary surgeon with final discretion to measure or not measure flow in the bypass graft.

Coronary anatomy and physiology represent a highly complicated and multifactorial patient-dependent system. On top of this, the addition of a bypass graft (saphenous vein graft or various arterial conduits) anastomosed to different coronary arteries in various configurations (in situ internal thoracic artery [ITA] vs a free graft anastomosed to the aorta or to the left ITA [LITA], with optional sequential grafting), along with an attempt to predict the patency of the coronary anastomosis downstream or the quality of the conduit bypassing the variable degrees of coronary stenoses based on flow parameters measured in the

From the Department of Cardiovascular Surgery, Mayo Clinic, Rochester, Minn. Disclosures: The author reported no conflicts of interest.

The Journal policy requires editors and reviewers to disclose conflicts of interest and to decline handling or reviewing manuscripts for which they may have a conflict of interest. The editors and reviewers of this article have no conflicts of interest.

Received for publication Feb 9, 2021; revisions received Feb 9, 2021; accepted for publication Feb 25, 2021; available ahead of print March 2, 2021.

Address for reprints: Chaim Locker, MD, Department of Cardiovascular Surgery, Mayo Clinic, 200 First St SW, Rochester, MN 55905 (E-mail: lekerlocker. chaim@mayo.edu).

JTCVS Techniques 2021;7:142-3

2666-2507

Copyright (c) 2021 The Authors. Published by Elsevier Inc. on behalf of The American Association for Thoracic Surgery. This is an open access article under the CC BY-NCND license (http://creativecommons.org/licenses/by-nc-nd/4.0/).

https://doi.org/10.1016/j.xjtc.2021.02.049

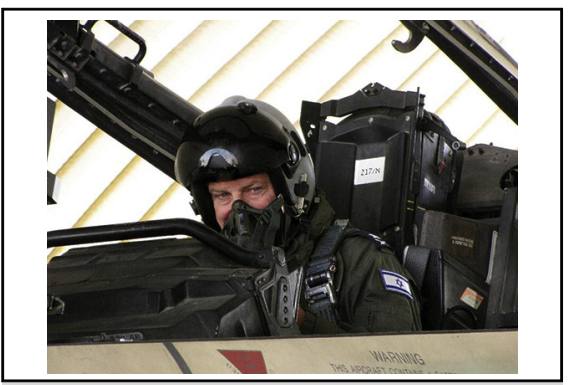

An $\mathrm{F}-15 \mathrm{I}$ pilot well trained in obligatory blind instrument-based flight.

CENTRAL MESSAGE

Intraoperative graft flow mea-

surement is not a recommenda-

tion but an incumbent obligation

for a coronary surgeon.

Although yet not perfect, cur-

rent instruments should un-

doubtedly be learned, trusted

and implemented for coronary

graft flow measurements.

conduit itself, is extremely ambitious, leaving aside the incremental complexity of the competitive flows phenomenon. A recent interesting study found that most transit-time flow measurement (TTFM) variables of the LITA to the left anterior descending artery (LAD) during CABG were strongly affected by the preoperative fractional flow reserve (FFR) values. ${ }^{3}$ The authors show that whereas minimum and mean graft flows were significantly negatively correlated with FFR, the pulsatility index and percentage insufficiency were significantly positively correlated with FFR. They recommended that because the fast Fourier transform ratio is not impacted by FFR, fast Fourier transform analysis of the TTFM should be performed in cases with an in situ LITA to the LAD with moderate stenosis and FFR $>0.75$. Without detailing the limitations of that study and arguing the authors' questionable recommendation, it definitely testifies to the complexity of the subject. Importantly, a major advantage of arterial coronary bypass grafting is in the superior coronary protection against future downstream atherosclerotic disease progression. ${ }^{4}$

In 2004, on commencing my advanced cardiovascular fellowship training program at the Mayo Clinic, I was surprised at the end of a CABG case to be required to measure the flows on each of the bypass grafts performed. This was 
the routine! Indeed, and with no enthusiasm, those flows were measured and charted. As a confident young arterial coronary surgeon, I doubted the need for those measurements. During the subsequent years of practice, I learned how important they are; apparently, more than a handful of patients owe their well-being to this simple routine.

What caused the pilot to crash his fighter jet into the ocean? There was no distress call, no attempt to eject, and no apparent aircraft malfunction. The pilot was very experienced, with 2300 hours of flight time. The investigation revealed that he experienced "unrecognized spatial disorientation," which caused him to misperceive altitude, position, and airspeed - in layperson's language, "pilot vertigo." Despite extensive training, experience, and technology, all based on knowledge of how flight affects human physiology, the pilot had no awareness that he was speeding downward to the ocean. The mandatory gyroscopic device in airplanes, invented in 1927, should have prevented it. "Flying blind," trusting the instruments, is against human instincts and skills, however. Pilots must learn against all contradictory sensations the difficult discipline of an absolute belief in their instruments. The same applies to coronary surgeons. We are obliged to trust our instruments and measurements, up to a level where if there is a doubt, then there is no doubt. If there is a questionable flow measurement, better consider it a red flag; do not trust your instincts, take the anastomosis down, reassess the conduit flow, revise the anastomosis, and then repeat the flow measurement. You will almost never regret revising a questionable bypass graft, but you may well regret going the other way. Indeed, and as noted by the authors, a recent study has shown that in $7.8 \%$ of CABG cases ( 1 out of 13 ) performed by very experienced surgeons, changes were made to the grafts as a consequence of the routine use of TTFM. ${ }^{5}$

Current knowledge and available instruments for measuring intraoperative bypass flows are not perfect and do not reveal the ultimate absolute conclusion required: should we or shouldn't we revise the bypass graft? This is an obstacle to implementing this mandatory routine in coronary surgery. It may well be that eventually more advanced analyses forms will need be involved (eg, artificial intelligence) to cope with the complexity of this subject, and the ability to create unanimously acceptable criteria for future intraoperative decisions related to optimal intravascular revascularization techniques. Notwithstanding, it is mandatory to create an organizational culture focused on quality assessment and safety measures in which coronary surgeons performing $\mathrm{CABG}$, especially but not limited to arterial grafting, are capable of and routinely obliged to be checking their outcomes on the spot by bypass graft flow measurement. ${ }^{6}$

With the current impressive technologic advances, with unmanned air vehicles and autonomous cars going uneventfully for thousands of miles, it is expected that medical and biotechnological companies should be able to generate an simple, operator-friendly flow measurement device that confirms and validates optimal CABG with highly accurate and trustable predictive values.

\section{References}

1. LeCompte T. The Disorient Express: despite the best training and technology, why do pilots still die from not knowing which end is up? Air \& Space Magazine. September 2008. Available at: https://www.airspacemag.com/military-aviation/ the-disorient-express-474780/. Accessed March 6, 2021.

2. Akhrass R, Bakaeen FG. Intraoperative graft patency validation: friend or foe? J Thorac Cardiovasc Surg Tech. 2021;7:131-7.

3. Noda M, Takami Y, Amano K, Sakurai Y, Akita K, Maekawa A, et al. Relation of fractional flow reserve with transit time coronary artery bypass graft flow measurement. Ann Thorac Surg. 2021;111:134-40.

4. Zhang M, Guddeti RR, Matsuzawa Y, Sara JDS, Kwon TG, Liu Z, et al. Left in ternal mammary artery versus coronary stents: impact on downstream coronary stenoses and conduit patency. J Am Heart Assoc. 2016;5:e003568.

5. Taggart DP, Thuijs DJFM, Di Giammarco G, Puskas JD, Wendt D, Trachiotis GD, et al. Intraoperative transit-time flow measurement and high-frequency ultrasound assessment in coronary artery bypass grafting. J Thorac Cardiovasc Surg. 2020; 159:1283-92.e2.

6. Gaudino MFL, Sandner S, Bonalumi G, Lawton JS, Fremes SE, Coronary Task Force of the European Association for Cardio-Thoracic Surgery. How to build a multi-arterial coronary artery bypass programme: a stepwise approach. Eur J Cardiothorac Surg. 2020;58:1111-7. 